\title{
THE GEOMETRY AND TOPOLOGY OF SINGLY-PERIODIC MINIMAL SURFACES *
}

\author{
WILLIAM H. MEEKS III $^{\dagger}$
}

1. Introduction. In 1993 Meeks and Rosenberg [8] proved that a properly embedded minimal surface $M$ in a complete flat nonsimply-connected three dimensional manifold $N$ has finite total curvature precisely when $M$ has finite topology. It follows from this result that such a surface $M$ is conformally equivalent to a finitely punctured compact Riemann surface $\bar{M}$ and that there is an analytic representation of $M$ in terms of meromorphic data on $\bar{M}$. Using this analytic representation, Meeks and Rosenberg characterized the asymptotic behavior of the annular ends of $M$ as being planar (asymptotic to a plane), Scherk (asymptotic to a half plane) or helicoidal (asymptotic to a helicoid) in the case the fundamental group of $N$ is cyclic. Under the assumption that $M$ is not flat, then it follows from the Strong Halfspace Theorem [2] that $M$ lifts to a connected properly embedded minimal surface $\tilde{M}$ in $\mathbb{R}^{3}$ invariant under a group $G$ of isometries which acts freely and discontinuously on $\mathbb{R}^{3}$ where $G$ is isomorphic to the fundamental group of $N$. In general, after taking a finite cover of $N$ and a homothetic scaling of the flat metric on $N$, we may assume that $N$ is isometric to a flat three-torus $\mathbb{T}^{3}, \mathbb{T} \times \mathbb{R}$ where $\mathbb{T}$ is a flat two-dimensional torus, $S^{1} \times \mathbb{R}^{2}$ where $S^{1}$ is the unit circle or $\mathbb{R}^{3} / S_{\theta}$ where $S_{\theta}$ is the screw motion symmetry which is the composition of the nontrivial rotation $R_{\theta}: \mathbb{R}^{3} \rightarrow \mathbb{R}^{3}$ counterclockwise around the $x_{3}$-axis by an angle $\theta, 0<\theta \leq \pi$, with the translation $\gamma\left(x_{1}, x_{2}, x_{3}\right)=\left(x_{1}, x_{2}, x_{3}+1\right)$. Note that in the above cases the fundamental groups of the possible quotient three-manifolds are free abelian groups of $\operatorname{rank} 3,2$, and 1 , respectively. We refer to a periodic minimal surface $\tilde{M}$ in $\mathbb{R}^{3}$ as being triply-periodic, doubly-periodic or singly-periodic depending on the rank of the fundamental group of these corresponding quotient spaces of $\mathbb{R}^{3}$. By the results of Meeks and Rosenberg, we have a very good understanding of periodic minimal surfaces in $\mathbb{R}^{3}$ whose quotients have finite topology by using the finite total curvature property. We refer the interested reader to [3], [7] and [8] for some nontrivial applications of these results.

In 1993 Meeks [4] obtained a topological obstruction for properly minimally embedding a surface with infinite topology into $\mathbb{T}^{2} \times \mathbb{R}$ by showing that such a surface must have a finite number of ends. This result and its proof motivated the recent theorem that the middle ends of a properly embedded minimal surface in $\mathbb{R}^{3}$ are never limit ends; this result implies that a properly embedded minimal surface of genus zero in $\mathbb{R}^{3}$ that is periodic has a quotient surface of finite topology and hence of finite total curvature. This last result, in turn, was used to prove the classification theorem of Meeks, Perez and Ros [5] that such a genus zero surface is either a plane, a helicoid or one of the Riemann examples which have an infinite number of planar ends.

In spite of the successes in restricting the topology of properly-embedded minimal surfaces in $\mathbb{T}^{2} \times \mathbb{R}, S^{1} \times \mathbb{R}$ and $\mathbb{R}^{3} / S_{\theta}$, still some rather bad situations can occur. For example, if one views one of Scherk's doubly-periodic minimal surfaces as a singlyperiodic minimal surface $M$ in an appropriately chosen quotient space which is $S^{1} \times \mathbb{R}^{2}$,

${ }^{*}$ Received November 28, 2002; accepted for publication June 10, 2003. This research was supported by NSF grant DMS - 0104044.

$\dagger$ Department of Mathematics and Statistics, University of Massachusetts, Amherst, MA 010039305, USA (bill@gang.umass.edu). 
then $M$ has genus zero with an infinite number of annular ends which are Scherk-type ends (note the similar result cannot occur in $\mathbb{T} \times \mathbb{R}$ by Meeks' theorem [4]). In this case $M$ has a single limit end which is the unique limit point in the natural topology on the space of ends of $M$. Also, there exist lifts of genus-one minimal surfaces in $\mathbb{T} \times \mathbb{R}$ to $S^{1} \times \mathbb{R}^{2}$ which as surfaces in $S^{1} \times \mathbb{R}^{2}$ have two limit ends and genus zero; the examples in $\mathbb{T} \times \mathbb{R}$ are described in [7] and have vertical parallel top and bottom ends. The surfaces just described in $S^{1} \times \mathbb{R}^{2}$ also have quotients in $\mathbb{R}^{3} / S_{\pi}$ which are Klein bottles with an infinite number of annular ends. If one is willing to consider surfaces of infinite genus, then there are orientable examples of properly embedded minimal surfaces with an infinite number of annular ends in in $\mathbb{R}^{3} / S_{\pi}$, which have one limit end (compare the existence of these examples with Theorems 1.1 and 1.2 and Conjecture 1.1 below); again, these examples can be chosen to be lifts of the same doubly-periodic minimal surfaces. The following theorem demonstrates that these topologically complicated surfaces cannot occur in the case $\mathbb{R}^{3} / S_{\theta}, \theta \neq \pi$.

THEOREM 1.1. If $M$ is a properly embedded minimal surface in $\mathbb{R}^{3} / S_{\theta}$ with $\theta \neq \pi$, then $M$ has a finite number of ends. Furthermore, if $M$ has at least two ends, then $M$ has quadratic area growth.

Our approach to proving Theorem 1.1 uses the analytic methods developed by Collin, Kusner, Meeks and Rosenberg [1] in their proof that the middle ends of a properly embedded minimal surface in $\mathbb{R}^{3}$ have quadratic area growth; a result which implies the middle ends are never limit ends. It should also be noted we will use this approach to give a new, simpler, and more analytic proof of the difficult technical "helicoidal" case of the finite total curvature theorem of Meeks and Rosenberg [8] for singly-periodic minimal surfaces. In particular, we give a proof, essentially independent from the results in [8], of the following theorem, a theorem which fails in $S^{1} \times \mathbb{R}^{2}$.

TheOREM 1.2. A properly embedded minimal surface in $\mathbb{R}^{3} / S_{\theta}, \theta \neq \pi$, has finite total curvature if and only if it has finite genus.

Based in part on Theorems 1.1 and 1.2, the author conjectures:

ConjeCture 1 . If $M \subset \mathbb{R}^{3} / S_{\theta}, \theta \neq \pi$, is a properly embedded minimal surface with at least two ends, then each end of $M$ is $C^{0}$-asymptotic to a plane, a vertical halfplane or a quotient of helicoid end. Furthermore, if $M \subset \mathbb{R}^{3} / S_{\pi}$ is orientable and has an infinite number of ends, then $M$ must also have infinite genus.

2. The proofs of the main theorems. We will first prove Theorem 1.1. Suppose $M \subset N=\mathbb{R}^{3} / S_{\theta 0}, \theta_{0} \neq \pi$, is a properly embedded minimal surface with more than one end. By solving Plateau-type problems in one of the complements of $M$ in $N$, it is straightforward to prove that there is a properly embedded stable noncompact minimal surface $\Sigma$ in $N-M$ with compact boundary and finite total curvature (see [6] for this type of construction). From the Weierstrass representation given in [8], Meeks and Rosenberg were able to show that $\Sigma$ could be chosen to be the end of a horizontal plane (planar-type), a vertical half annulus (Scherk-type), or a quotient of an end of a helicoid (helicoidal-type). In each of these cases we may assume that $\Sigma$ lies outside a fixed open cylinder $C \subset N$ with axis the quotient of the $x_{3}$-axis and such that $\partial \Sigma \subset \partial C$. We will say that $M$ has planar-type, Scherk-type or helicoidal type respectively, if $\Sigma$ has this type; this makes sense since if $M$ has an associated $\Sigma$ of one such type, then any other $\Sigma^{\prime}$ also has the same type. 
In the case $M$ has planar-type, as we mentioned in the Introduction, the results in [1] imply that $M$ has quadratic area growth and a finite number of ends. In the case $M$ has Scherk-type, a slight modification of the proof of the theorem of Meeks [4] that a properly embedded minimal surface with compact boundary in $S^{1} \times[0, L] \times \mathbb{R}$ has linear area growth shows that $M$ has linear area growth, which implies that $M$ has at most quadratic area growth. It remains to consider the case where $M$ is of helicoidal-type.

For convenience of notation, we will let $M$ also denote the properly embedded minimal surface $M-C$ with compact boundary contained in the three manifold $\bar{N}$ which is the geodesic closure of $N-(C \cup \Sigma)$. Since the fundamental group of $\bar{N}$ is $\mathbb{Z}$, the functions $\theta$ and $x_{3}$ are well-defined up to integer multiples of respective constants in the flat three manifold $\bar{N}$ and hence $c x_{3}-\theta$ is well-defined for some nonzero constant $c$. Hence, after a fixed homothety of $\bar{N}$ by $\frac{1}{c}$, we will from now on assume that $x_{3}-\theta$ is well-defined.

The following lemma immediately follows from the calculations in the proof of Lemma 2.2 in [1].

LeMma 2.1. Let $M \subset \bar{N}$ be as described above. Let $r=\sqrt{x_{1}^{2}+x_{2}^{2}}$ and $g: M \rightarrow$ $\mathbb{C} \cup\{\infty\}$ be the locally well-defined Gauss map for $M$ in the flat three-manifold $\bar{N}$ composed with stereographic projection to $\mathbb{C} \cup\{\infty\}$. Then at the points where $\nabla x_{3} \neq 0$, we have

$$
\Delta \ln r=\operatorname{Re}\left(\frac{\mathrm{g}}{\overline{\mathrm{g}}} \cdot \frac{\left|\nabla \mathrm{x}_{3}\right|^{2}}{\mathrm{r}^{2} \mathrm{e}^{2 \mathrm{i} \theta}}\right)
$$

and

$$
\Delta \theta=\operatorname{Im}\left(\frac{\mathrm{g}}{\overline{\mathrm{g}}} \cdot \frac{\left|\nabla \mathrm{x}_{3}\right|^{2}}{\mathrm{r}^{2} \mathrm{e}^{2 \mathrm{i} \theta}}\right) .
$$

In particular, $|\Delta \ln r| \leq\left|\nabla x_{3}\right|^{2} / r^{2}$ and $|\Delta \theta| \leq\left|\nabla x_{3}\right|^{2} / r^{2}$.

Proof of Theorem 1.1. Lemma 2.2 in [1] was used to prove that the middle ends of a properly embedded minimal surface in $\mathbb{R}^{3}$ have quadratic area growth. In our proof of Theorem 1.1 we will follow closely the arguments of the similar theorem on quadratic area growth of middle ends in [1] which used the estimate $|\Delta \ln r| \leq$ $\left|\nabla x_{3}\right|^{2} / r^{2}$. However, here the needed estimates are more delicate and we will be forced to use the explicit formulas in Lemma 2.2 for both $\Delta \ln r$ and $\Delta \theta$ given in terms of the locally defined Gauss map $g$. We now proceed with the proof of Theorem 1.1 .

Let $C_{t}=\left\{p \in \mathbb{R}^{3} \mid r(p)=t\right\}$ be the vertical cylinder of radius $t$ and let $M_{t}$ be the part of $M$ inside $C_{t}$. Since the part of $M$ inside the geodesic ball of radius $R$ centered at the origin is contained in $M_{R}$, it suffices to prove that $M_{R}$ has quadratic area growth.

In the complement of the $x_{3}$-axis, one has the orthonormal basis for $\mathbb{R}^{3}$ : $\left(\bar{\nabla} r, \bar{\nabla} x_{3}, r \bar{\nabla} \theta\right)=\left(A_{1}, A_{2}, A_{3}\right)$. Let $B_{i}=A_{i}-\left(\vec{n} \cdot A_{i}\right) \vec{n}$ be the tangent part of $A_{i}$ $(\vec{n}=$ the unit normal to $M)$, so

$$
\left|B_{i}\right|^{2}=\left|A_{i}\right|^{2}-\left(\vec{n} \cdot A_{i}\right)^{2}=1-\left(\vec{n} \cdot A_{i}\right)^{2} .
$$

Hence, $\left|B_{1}\right|^{2}+\left|B_{2}\right|^{2}=1+\left(\vec{n} \cdot A_{3}\right)^{2} \geq 1$. Since $B_{1}=\nabla_{M} r$ and $B_{2}=\nabla_{M} x_{3}$,

$$
\left|\nabla_{M} r\right|^{2}+\left|\nabla_{M} x_{3}\right|^{2} \geq 1 \text {. }
$$


Thus,

$$
\int_{M_{R}} d A \leq \int_{M_{R}}\left(\left|\nabla_{M} r\right|^{2}+\left|\nabla_{M} x_{3}\right|^{2}\right) d A
$$

Therefore, it remains to prove that both $\int_{M_{R}}\left|\nabla_{M} r\right|^{2} d A$ and $\left.\int_{M_{R}}\left|\nabla_{M} x_{3}\right|^{2}\right) d A$ grow at most quadratically in $R$. Henceforth we will drop the subscript of $M$ from $\nabla_{M}$.

Now consider the function $h: M \rightarrow R$ defined by $h=\ln r-\left(x_{3}-\theta\right)^{2}$. Since $\left(x_{3}-\theta\right)^{2}$ is actually a bounded function on $\bar{N}, h$ is a proper function on $M$. By chosing $C$ sufficiently large, we may assume that $h$ is also positive on $M$. We now check that $\Delta h \leq 0$ on $M$ when the cylinder $C$ is chosen sufficiently large.

Since $x_{3}-\theta$ is bounded, Lemma 2.1 implies that $\left|\Delta \ln r-2\left(x_{3}-\theta\right) \Delta \theta\right| \leq \frac{c_{0}\left|\nabla x_{3}\right|^{2}}{r^{2}}$ for some constant $c_{0}$. Note that

$\Delta h=\Delta \ln r+2\left(x_{3}-\theta\right) \Delta \theta-2\left|\nabla x_{3}-\nabla \theta\right|^{2}$. Since $\|v-w\| \geq|\|v\|-\|w\||$ and $|\nabla \theta| \leq \frac{1}{r}$, then, for any small $\varepsilon>0$ and $C$ sufficiently large, $\left|\nabla x_{3}-\nabla \theta\right| \geq \varepsilon\left|\nabla x_{3}\right|$ whenever $\left|\nabla x_{3}\right|>\frac{1+\varepsilon}{r}$ or $\left|\nabla x_{3}\right|<\frac{1-\varepsilon}{r}$. On the other hand, for $\left|\nabla x_{3}\right| \neq 0, \varepsilon>0$ small, $r$ large and $\left|\nabla x_{3}-\nabla \theta\right|<\varepsilon\left|\nabla x_{3}\right|$, then $\left|\nabla x_{3}\right|$ and $|\nabla \theta|$ are each approximately $\frac{1}{r}$ and argument of the complex number $\frac{g}{\bar{g} e^{2 i \theta}}$ is approximately $\pi$. But when the argument of $\frac{g}{\bar{g} e^{2 i \theta}}$ is sufficiently close to $\pi$, then a straightforward calculation using the formulas for $\Delta \ln r$ and $\Delta \theta$ given in Lemma 2.1 shows that $\Delta \ln r+2\left(x_{3}-\theta\right) \Delta \theta$ is nonpositive and has absolute value approximately equal to $\frac{\left|\nabla x_{3}\right|^{2}}{r^{2}}$. Hence, we now may assume that for some $c_{1}>0, \Delta h \leq-c_{1}\left|\nabla x_{3}\right|^{2}$ for $c_{1}$ chosen sufficiently small.

Now for any proper positive $C^{2}$-function $H$ on $M, \Delta H \leq 0$, and $T \geq \sup (h(\partial M))$, the divergence theorem implies

$$
\int_{H^{-1}([0, T])} \Delta H=-\int_{\partial M} \nabla H \cdot \nu+\int_{H^{-1}(T)}|\nabla H|,
$$

where $\nu$ is the outward pointing conormal to the boundary. Thus, we have $\int_{H^{-1}(T)}|\nabla H|$ is positive monotonically decreasing as $T \rightarrow \infty$. Hence, $\Delta H \in L^{1}(M)$. By letting $H=h$, we see that $\Delta h$ is in $L^{1}(M)$.

We now prove that $\int\left|\nabla x_{3}\right|^{2}$ grows quadratically. Since $\Delta h \leq-c_{1} \frac{\left|\nabla x_{3}\right|^{2}}{r^{2}}$ and $\Delta h \in L^{1}(M), \int_{M} \frac{\left|\nabla x_{3}\right|^{2}}{r^{2}}<c_{2}$ some constant $c_{2}$. Therefore, $\int_{M_{R}} \frac{\left|\nabla x_{3}\right|^{2}}{r^{2}} \leq c_{2}$ on $M_{R}$. Since $r \leq R, \int_{M_{R}}\left|\nabla x_{3}\right|^{2} \leq c_{2} R^{2}$ which proves that $\int_{M_{R}}\left|\nabla x_{3}\right|^{2}$ grows at most quadratically in $R$.

Just as we showed that $h=\ln r-\left(x_{3}-\theta\right)^{2}$ is a proper and positive superharmonic function on $M$ outside of some compact subset of $M$ and $\Delta h \in L^{1}(M)$, we also see that $\Delta(h+\ln r) \in L^{1}(M)$. Since $\Delta h$ and $\Delta h+\Delta \ln r$ are both in $L^{1}(M)$, it follows that $\Delta \ln r \in L^{1}(M)$.

Since

$$
\int_{M_{R}} \Delta_{M} \ln r=-\int_{\partial M} \frac{\nabla_{M} r \cdot \nu}{r}+\int_{C_{R} \cap M} \frac{\left|\nabla_{M} r\right|}{R}=c_{3}+\frac{1}{R} \int_{C_{R} \cap M}\left|\nabla_{M} r\right|
$$

and $\int_{M_{R}}|\Delta \ln r|$ converges, $\frac{1}{R} \int_{C_{R} \cap M}\left|\nabla_{M} r\right|$ has a finite limit as $R \rightarrow \infty$. Hence, $\int_{C_{R} \cap M}\left|\nabla_{M} r\right| \leq c_{4} R$ for some constant $c_{4}$.

Since

$$
\int_{C_{r} \cap M}\left|\nabla_{M} r\right| \leq c_{4} r
$$


the coarea formula implies

$$
\int_{M_{R}}\left|\nabla_{M} r\right|^{2} \leq \int_{1}^{R} c_{4} r d r \leq \frac{c_{4} R^{2}}{2}
$$

which means that $\int_{M_{R}}\left|\nabla_{M} r\right|^{2}$ grows quadratically in $R$. It now follows that the area of $M$ grows quadratically in $R$.

We now check that $M$ has a finite number of ends. If $E$ is a proper noncompact subdomain of $M$ with $\partial E$ compact, then we know that the area of $E$ grows at most quadratically. But, by the monotonicity formula for area [9], the area of $E$ must grow asymptotically at least as quickly as a fixed fraction $c$ of the area of a plane (this fraction is $\frac{\theta_{0}}{2 \pi}$ ), which means that for large $R$ the area of $E$ inside the ball $B(R)$ is at least $c \pi R^{2}$. If $M$ has at least $n$ ends, then the area growth of $M$ cannot be less than $n c \pi R^{2}$. In particular, the last sentence in the statement of Theorem 1.1 holds, which completes our proof.

Proof of Theorem 1.2. Suppose now that $M$ is a properly embedded minimal surface in $\mathbb{R}^{3} / S_{\theta}, \theta \neq \pi$, with finite genus. If $M$ has more than one end, then, by Theorem 1.1, $M$ has quadratic area growth and a finite number of annular ends. However, when $M$ has finite genus and one end, this end is an annular end. In this case it is straightforward to prove, using that the fundamental group of the end is cylic, that there exists an end of a plane, an end of a helicoid or a vertical flat halfannulus (Scherk end) in $\mathbb{R}^{3} / S_{\theta}-M$, which is all that is needed to prove that $M$ has quadratic area growth. However, if $M$ is any complete Riemannian surface with nonpositive Gaussian curvature and intrinsic area growth less than $c R^{2}$, where $R$ is the distance from a fixed point, then the total curvature of $M$ is greater than or equal to $-2 c+2 \pi \chi(M)$ where $\chi(M)$ is the Euler characteristic of $M$. Hence, $M$ has finite total curvature, which completes the proof of Theorem 1.2.

REMARK 2.2. One should note that if $M$ is a properly embedded minimal surface in $S^{1} \times \mathbb{R}^{2}$ or $\mathbb{R}^{3} / S_{\theta}$ and $M$ is of helicoidal type or planar type, then the arguments given in the proof of Theorem 1.1 imply that $M$ has quadratic area growth and a finite number of ends.

\section{REFERENCES}

[1] P. Collin and R. Kusner and Meeks III, W. H. And H. RosenberG, The geometry, conformal structure and topology of minimal surfaces with infinite topology, preprint.

[2] D. HofFman AND MEeKs III, W. H., The strong halfspace theorem for minimal surfaces, Invent. Math., 101 (1990), pp. 373-377.

[3] H. LAZARD-Holly AND MeEKS III, W. H., The Classification of embedded doubly-periodic minimal surfaces of genus zero, Invent. Math., 143 (2001), pp. 1-27.

[4] MeEks III, W. H., The geometry, topology, and existence of periodic minimal surfaces, Proceedings of Symposia in Pure Math., 54 (1993), pp. 333-374, Part I.

[5] MeEks III, W. H. AND J. PÉREz AND A. Ros, Uniqueness of the Riemann minimal examples, Invent. Math., 131 (1998), pp. 107-132.

[6] MEeks III, W. H. AND H. Rosenberg, Minimal surfaces of finite topology, preprint.

[7] MeEks III, W. H. AND H. RosenBerG, The global theory of doubly periodic minimal surfaces, Invent. Math., 97 (1989), pp. 351-379.

[8] Meeks III, W. H. AND H. RosenberG, Singly periodic minimal surfaces, Comment. Math. Helvetici, 68 (1993), pp. 538--578.

[9] L. Simon, Lectures on Geometric Measure Theory, in Proceedings of the Center for Mathematical Analysis, volume 3, Canberra, Australia, 1983. Australian National University. 
